\title{
Adaptive Time-Space Algorithms for the Simulation of Multi-scale Reaction Waves
}

Max Duarte, Marc Massot, Stéphane Descombes and Thierry Dumont

\begin{abstract}
We present a new resolution strategy for multi-scale reaction waves based on adaptive time operator splitting and space adaptive multiresolution, in the context of localized and stiff reaction fronts. The main goal is to perform computationally efficient simulations of the dynamics of multi-scale phenomena under study, considering large simulation domains with conventional computing resources. We aim at time-space accuracy control of the solution and splitting time steps purely dictated by the physics of the phenomenon and not by stability constraints associated with mesh size or source time scales. Numerical illustrations are provided for $2 \mathrm{D}$ and $3 \mathrm{D}$ combustion applications modeled by reaction-convection-diffusion equations.
\end{abstract}

Key words: time adaptive integration, space adaptive multiresolution, combustion MSC2010: 65M08, 65M50, 65Z05, 65G20

\section{Introduction}

Numerical simulations of multi-scale phenomena are commonly used for modeling purposes in many applications such as combustion, chemical vapor deposition, or air pollution modeling. In general, all these models raise several difficulties created by the high number of unknowns, the wide range of temporal scales due to large

M. Duarte, M. Massot

Laboratoire EM2C - UPR CNRS 288, Ecole Centrale Paris, Grande Voie des Vignes, 92295

Chatenay-Malabry Cedex, France, e-mail: $\{\max$.duarte, marc.massot $\}$ @em $2 \mathrm{c}$.ecp. fr

S. Descombes

Laboratoire J. A. Dieudonné - UMR CNRS 6621, Université de Nice - Sophia Antipolis, Parc Valrose, 06108 Nice Cedex 02, France, e-mail: sdescomb@unice.fr

T. Dumont

Institut Camille Jordan - UMR CNRS 5208, Université de Lyon, 43 Boulevard du 11 novembre 1918, 69622 Villeurbanne Cedex, France, e-mail: tdumont @math. univ-lyon 1 . fr 
and detailed chemical kinetic mechanisms, as well as steep spatial gradients associated with localized fronts of high chemical activity. In this context, faced with the induced stiffness of these time dependent problems, a natural stumbling block to perform 3D simulations with all scales resolution is either the unreasonably small time step due to stability requirements or the unreasonable memory and computing time required by implicit methods. Furthermore, an accurate description of such spatial multi-scale phenomena would also lead to large and sometimes unfeasible computation domains, if no adaptive meshing technique is used.

To overcome these difficulties, we present a new numerical strategy with a time operator splitting that considers dedicated high order time integration methods for reaction, diffusion and convection problems, in order to build a time operator splitting scheme that exploits efficiently the special features of each problem. Based on recent theoretical studies of numerical analysis, such a strategy leads to a splitting time step which is not restricted neither by the fastest scales in the source term nor by restrictive stability limits of diffusive or convective steps, but only by the physics of the phenomenon. Moreover, this splitting time step is dynamically adapted taking into account local error estimates [4]. The time integration is performed over a dynamic adapted grid obtained by multiresolution techniques in a finite volumes framework $[9,2,11]$, which on the one hand, yield important savings in computing resources and on the other hand, allow to somehow control the spatial accuracy of the compressed representation based on a solid mathematical background.

Even though, the strategy was developed for the resolution of general multi-scale phenomena in various domains as biomedical applications [7] or nonlinear chemical dynamics [6], we will focus here on multidimensional combustion problems at large Reynolds numbers in order to assess the capability of the method. The paper is organized as follows: section 2 describes briefly the numerical strategy, based on spatial adaptive multiresolution and second order adaptive time integration. Physical configuration and modeling equations are presented in section 3 for laminar premixed flames interacting with vortices, along with $2 \mathrm{D}$ and $3 \mathrm{D}$ numerical illustrations. We end in the last part with some concluding remarks.

\section{Construction of the Numerical Strategy}

We detail briefly the developed operator splitting strategy with splitting time step adaptation, and some fundamental aspects of the adaptive multiresolution method.

\subsection{Adaptive Time Operator Splitting}

Given a general convection-reaction-diffusion system of equations

$$
\partial_{t} \mathbf{u}-\partial_{\mathbf{x}}\left(\mathbf{F}(\mathbf{u})+\mathbf{D}(\mathbf{u}) \partial_{\mathbf{x}} \mathbf{u}\right)=\mathbf{f}(\mathbf{u}), \quad \mathbf{x} \in \mathbb{R}^{d}, t>0,
$$


with $\mathbf{u}(0, \mathbf{x})=\mathbf{u}_{0}(\mathbf{x})$, where $\mathbf{F}, \mathbf{f}: \mathbb{R}^{m} \rightarrow \mathbb{R}^{m}$ and $\mathbf{u}: \mathbb{R} \times \mathbb{R}^{d} \rightarrow \mathbb{R}^{m}$, with diffusion matrix $\mathbf{D}(\mathbf{u})$ : a tensor of order $d \times d \times m$; an operator splitting procedure allows to consider dedicated solvers for the reaction part which is decoupled from the other physical phenomena like convection, diffusion or both, for which there also exist dedicated numerical methods. These dedicated methods chosen for each subsystem are then responsible for dealing with the fast scales associated with each one of them, in a separate manner, while the reconstruction of the global solution by the splitting scheme should guarantee an accurate description with error control of the global physical coupling, without being related to the stability constraints of the numerical resolution of each subsystem.

A second order Strang scheme is then implemented [12]

$$
\mathscr{S}^{\Delta t}\left(\mathbf{u}_{0}\right)=\mathscr{R}^{\Delta t / 2} \mathscr{D}^{\Delta t / 2} \mathscr{C}^{\Delta t} \mathscr{D}^{\Delta t / 2} \mathscr{R}^{\Delta t / 2}\left(\mathbf{u}_{0}\right),
$$

where operators $\mathscr{R}, \mathscr{D}, \mathscr{C}$ indicate respectively the independent resolution of the reaction, diffusion and convection problems with $\Delta t$ defined as the splitting time step. Usually, for propagating reaction waves where for instance, the speed of propagation is much slower than some of the chemical scales, the fastest scales are not directly related to the global physics of the phenomenon, and thus, larger splitting time steps might be considered. Nevertheless, order reductions may then appear due to shortlife transients associated to fast variables and in these cases, it has been proved in [5] that better performances are expected while ending the splitting scheme by operator $\mathscr{R}$ or in a more general case, the part involving the fastest time scales of the phenomenon.

An adaptive splitting time step strategy, based on a local error estimate at the end of each $\Delta t$, is implemented in order to control the accuracy of computations. A second, embedded and lower order Strang splitting method $\widetilde{\mathscr{S}}^{\Delta t}$ was developed [4] in order to dynamically calculate a local error estimate that should verify

$$
\left\|\mathscr{S}^{\Delta t}\left(\mathbf{u}_{0}\right)-\widetilde{\mathscr{S}}^{\Delta t}\left(\mathbf{u}_{0}\right)\right\| \approx \mathscr{O}\left(\Delta t^{2}\right)<\eta_{\text {split }}
$$

in order to accept current computation with $\Delta t$, and thus, the new splitting time step is given by

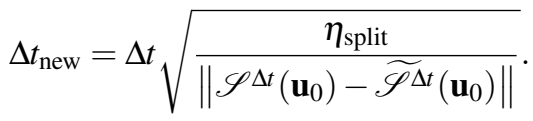

The choice of suitable time integration methods to approximate numerically $\mathscr{R}$, $\mathscr{D}$ and $\mathscr{C}$ during each $\Delta t$ is mandatory not only to guarantee the theoretical framework of the numerical analysis but also to take advantage of the particular features of each independent subproblem. A new operator splitting for reaction-diffusion systems was recently introduced [6], which considers a high fifth order, $A$-stable, $L$-stable method like Radau5 [8], based on implicit Runge-Kutta schemes for stiff ODEs, that solves with a local cell by cell approach the reaction term: a system of stiff ODEs without spatial coupling. On the other hand, a high fourth order method was chosen, like ROCK4 [1], based on explicit stabilized Runge-Kutta schemes 
which features extended stability domains along the negative real axis, very appropriate for diffusion problems because of the usual predominance of negative real eigenvalues. Both methods incorporate adaptive time integration tools, similar to (4), in order to control accuracy for given $\eta_{\text {Radaus }}$ and $\eta_{\text {ROCK4 }}$.

An explicit high order in time and space one step monotonicity preserving scheme OSMP [3] is used as convective scheme. It combines monotonicity preserving constraints for non-monotone data to avoid extrema clipping, with TVD features to prevent spurious oscillations around discontinuities or sharp spatial gradients. Classical CFL stability restrictions are though imposed during each splitting time step $\Delta t$. Notice that the overall combination of explicit treatment of spatial phenomena as convection and diffusion, with local implicit integration of stiff reaction implies important savings in computing time and memory resources. For the reaction, local treatment plus adaptive time stepping allow to discriminate cells of high reactive activity in the neighborhood of the localized wavefront, saving as a consequence a large quantity of integration time.

\subsection{Mesh Refinement Technique}

We are concerned with the propagation of reacting wavefronts, hence important reactive activity as well as steep spatial gradients are localized phenomena. This implies that if we consider the resolution of reactive problem, a considerable amount of computing time is spent on nodes that are practically at (partial) equilibrium. Moreover, there is no need to represent these quasi-stationary regions with the same spatial discretization needed to describe the reaction front, so that convection and diffusion problems might also be solved over a smaller number of nodes. An adapted mesh obtained by a multiresolution process which discriminates the various space scales of the phenomenon, turns out to be a very convenient solution to overcome these difficulties $[6,7]$.

In practice, if one considers a set of nested spatial grids from the coarsest to the finest one, a multiresolution transformation allows to represent a discretized function as values on the coarsest grid plus a series of local estimates at all other levels of such nested grids. These estimates correspond to the wavelet coefficients of a wavelet decomposition obtained by inter-level transformations, and retain the information on local regularity when going from a coarse to a finer grid. Hence, the main idea is to use the decay of the wavelet coefficients to obtain information on local regularity of the solution: lower wavelet coefficients are associated to local regular spatial configurations and vice-versa. This representation yields to a thresholding process that builds dynamically the corresponding adapted grid on which the solutions are represented; then the error committed by the multiresolution transformation is proportional to $\eta_{\mathrm{MR}}$, where $\eta_{\mathrm{MR}}$ is a threshold parameter $[9,2]$. 


\section{Numerical Illustration}

In these illustrating examples, we are concerned with the numerical simulation of premixed flames interacting with vortex structures: a pair of counter rotating vortices in a $2 \mathrm{D}$ configuration and a 3D toroidal vortex. This is usually a difficult problem to solve because of the localized and stiff reactive fronts, even more with large Reynolds numbers. Nevertheless, in order to properly evaluate the proposed strategy we consider only time evolution problems for which the hydrodynamics is not solved but a large Reynolds number velocity field is imposed. Based on a model presented in [10], we consider that the chemistry may be modeled by a global, single step, irreversible reaction characterized by an Arrhenius law; and a thermodiffusive approach of laminar flame theory is adopted in order to decouple velocity field computation from determination of species mass fractions and temperature. Known solutions of incompressible Navier-Stokes equations may then be imposed, and the problem is reduced to solving the standard species and energy balance equations.

Following [10], a progress variable $c(x, y, t)$ is introduced:

$$
c=\frac{T-T_{\mathrm{o}}}{T_{\mathrm{b}}-T_{\mathrm{o}}}
$$

where subscripts ()$_{\mathrm{o}}$ and ()$_{\mathrm{b}}$ indicate respectively, fresh mixture zone and burnt product zone; and we finally obtain for a 2D configuration

$$
\frac{\partial c}{\partial t_{\star}}+u_{\star} \frac{\partial c}{\partial x_{\star}}+v_{\star} \frac{\partial c}{\partial y_{\star}}-\left(\frac{\partial^{2} c}{\partial x_{\star}^{2}}+\frac{\partial^{2} c}{\partial y_{\star}^{2}}\right)=\operatorname{Da}(1-c) \exp \left(-\frac{T_{\mathrm{a}}}{T_{\mathrm{o}}(1+\tau c)}\right),
$$

where Da is a Damköhler number, $T_{\mathrm{a}}$ the activation energy, $\tau=T_{\mathrm{b}} / T_{\mathrm{o}}-1$, and ()$_{\star}$ indicates dimensionless variables. The velocity field $\left(u_{\star}(t), v_{\star}(t)\right)$ is deduced analytically and imposed into (6), considering a $2 \mathrm{D}$ viscous core vortex with a dimensionless azimuthal velocity of the form:

$$
v_{\theta \star}\left(r_{\star}, t_{\star}\right)=\frac{\operatorname{ReSc}}{r_{\star}}\left(1-\exp \left(-\frac{r_{\star}^{2}}{4 \operatorname{Sc} t_{\star}}\right)\right),
$$

with $r_{\star}\left(x_{\star}, y_{\star}\right)$, the distance to the vortex center, Reynolds and Schmidt numbers.

Figure 1 shows the interaction of the premixed flame with two counter rotating vortices modeled each one of them by (7), centered at $(-0.25,-0.5)$ and $(0.25,-0.5)$ for a $2 \mathrm{D}$ spatial domain of $[-1,1]^{2}$. The upper (red) and lower (blue) regions correspond respectively to burnt product $(c=1)$ and fresh mixture $(c=0)$ zones. The corresponding adapted mesh tightens around the stiff regions and propagates along the wavefronts.

The following modeling values were considered into (6) and (7): $\mathrm{Da}=2.5 \times$ $10^{9}, T_{\mathrm{a}}=20000 \mathrm{~K}, T_{\mathrm{o}}=300 \mathrm{~K}, T_{\mathrm{b}}=2315.4 \mathrm{~K}, \tau \approx 6.72, \mathrm{Sc}=1$ and $\mathrm{Re}=1000$. The initial condition corresponds to a planar premixed flame at $y=-0.5$ and the phenomenon is studied over a time domain of $\left[0,4 \times 10^{-3}\right]$. The MR procedure considers a set of 10 grids, equivalent to $1024^{2}=1048576$ cells on the finest grid. 


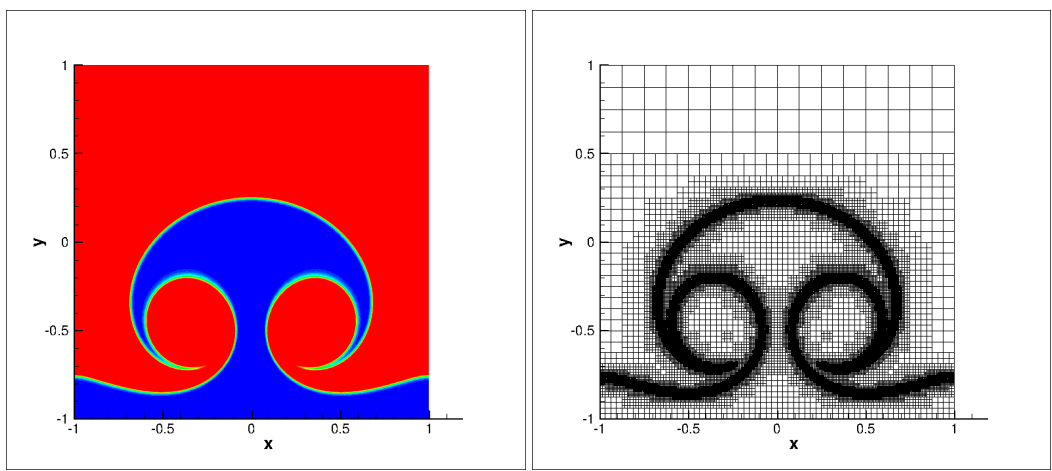

Fig. 12 D premixed flame interacting with two counter rotating vortices. Solution of variable $c$ at $t_{\star}=4 \times 10^{-4}$ (left) and corresponding adapted mesh (right). Finest grid: $1024^{2}$

MR and adaptive splitting time step tolerances were set to $\eta_{\mathrm{MR}}=10^{-2}$ and $\eta_{\text {split }}=$ $10^{-3}$, with $\eta_{\text {Radau } 5}=\eta_{\text {ROCK4 }}=10^{-5}$.
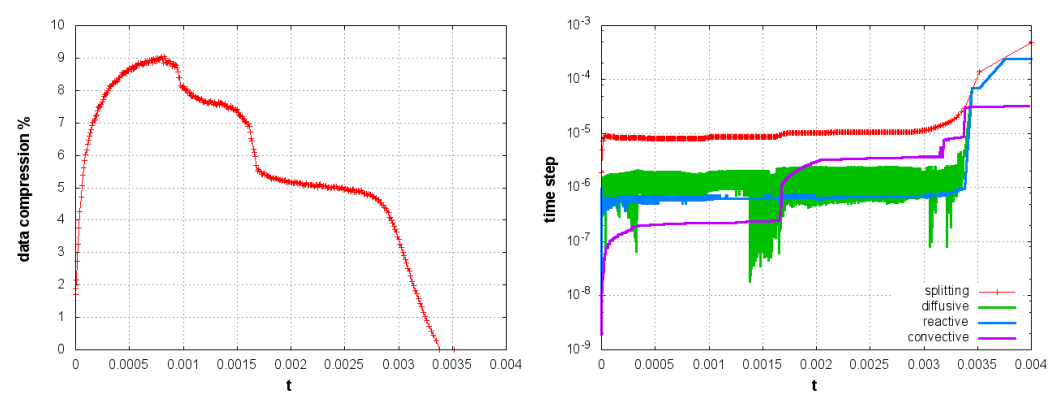

Fig. 2 2D premixed flame interacting with two counter rotating vortices. Time evolution of data compression in the solution representation (left) and splitting, diffusive, reactive and convective time steps (right). Finest grid: $1024^{2}$

Figure 2 shows data compression obtained by MR representation of the solution, measured as the percentage of active cells with respect to the finest grid representation; in this case, lower than $9 \%$ of $1024^{2}$. On the other hand, splitting time step starts from an initial value set to $10^{-8}$ in order to handle correctly the initial sudden apparition of the vortices, that evolves rapidly to a final quasi stable value of $10^{-5}$, which indicates the decoupling degree achieved within the accuracy prescribed to describe the global propagating phenomenon. The corresponding convective time step with CFL $=1$ illustrates the time scale decoupling obtained by a splitting technique and highlights the eventual inconveniences of solving (6) considering all phenomena at once. The same conclusion is valid concerning reactive and diffusive time steps. By the way, larger convective time steps are used thanks to the adapted 
grid representation which allows to discriminate locally large velocity values (in this case $\left|u_{\star}\right|,\left|v_{\star}\right| \approx 40000$ ) from the refined regions around the wavefront, as we can see in the "jumps" of convective time steps in Fig. 2. Reactive time steps correspond to cells at the wavefront (for furthest cells, reactive time steps are equal to splitting ones), while lower diffusive time steps are needed in order to fulfill each splitting time step, which explains the "oscillations". Diffusive time steps might take values beyond classical stability constraints (of the order of $10^{-6}$ for explicit RK4 [8] and eigenvalues of $-2.2 \times 10^{6}$ ), and it is finally set by the accuracy criterion.

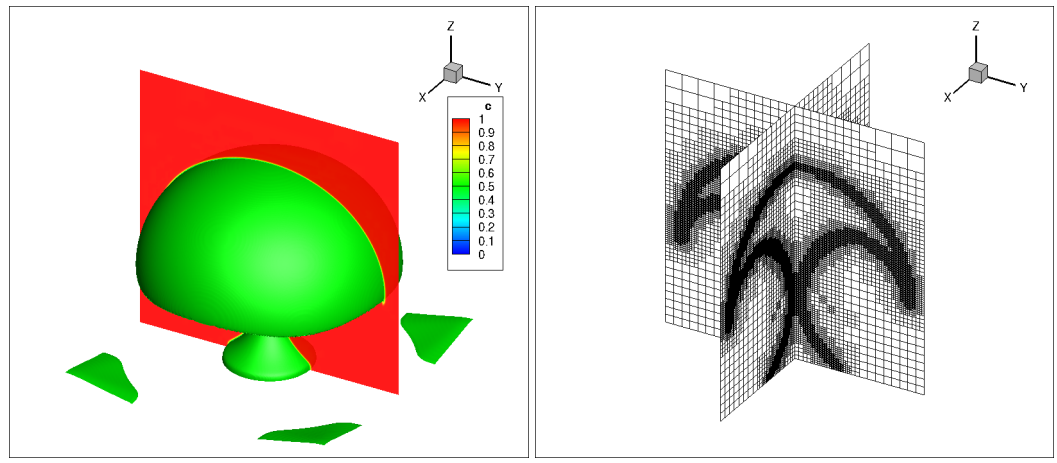

Fig. 3 3D premixed flame interacting with a toroidal vortex. Solution of variable $c$ at $t_{\star}=1.1 \times$ $10^{-3}$ showing isosurface $c=0.5$ (left) and corresponding adapted mesh (right). Finest grid: $256^{3}$

This resolution technique has a straightforward extension to 3D configurations. Figure 3 shows the interaction of the premixed flame with a toroidal vortex modeled by (7) centered at $\sqrt{x_{\star}^{2}+y_{\star}^{2}}=0.25, z_{\star}=-0.5$ for a $3 \mathrm{D}$ spatial domain of $[-1,1]^{3}$. The modeling and tolerance parameters are taken equal to the 2D case and the MR procedure considers a set of 8 grids, equivalent to $256^{3}=16777216$ cells on the finest grid. The splitting time step shows the same behavior as for the previous case with same order of values, while the data compression is lower than $17 \%$, taking into account that a lower scale discrimination is available with 8 different grids. All the computations have been performed on a AMD Shanghai processor of $2.7 \mathrm{GHz}$ with memory capacity of $4 \mathrm{~GB}$. Computing times for the 2D and 3D configurations were about of $0 \mathrm{~h} 57 \mathrm{~m}$ and $14 \mathrm{~h} 40 \mathrm{~m}$, respectively.

\section{Concluding Remarks}

The present work proposes a new numerical approach which is shown to be computationally efficient. It couples adaptive multiresolution techniques with a new operator splitting strategy with high order time integration methods to properly solve the entire spectrum of scales of each phenomenon. The splitting time step is cho- 
sen on the sole basis of the structure of the continuous system and its decoupling capabilities, but not related to stability requirements of the numerical methods involved in order to integrate each subsystem, even if stiffness is present. The global accuracy of the simulation is controlled and dynamically evaluated based on theoretical and numerical results. As a consequence, the resulting highly compressed data representations as well as the accurate and feasible resolution of these stiff phenomena prove that large computational domains previously out of reach can be successfully simulated with conventional computing resources. At this stage of development, the same numerical strategy can be coupled to a hydrodynamics solver, considering though that an important amount of work is still in progress concerning programming features such as data structures and parallelization strategies.

Acknowledgements This research was supported by fundamental project grants from ANR Séchelles (project leader S. Descombes), and DIGITEO MUSE (project leader M. Massot). M. Duarte has a Ph.D. grant from Mathematics (INSMI) and Engineering (INSIS) Institutes of CNRS and supported by INCA project. Authors express special thanks to Christian Tenaud (LIMSICNRS) for providing the basis of the multiresolution kernel of MR CHORUS (DI 03760-01).

\section{References}

1. Abdulle, A.: Fourth order Chebyshev methods with recurrence relation. SIAM J. Sci. Comput. 23, 2041-2054 (2002)

2. Cohen, A., Kaber, S., Müller, S., Postel, M.: Fully adaptive multiresolution finite volume schemes for conservation laws. Math. of Comp. 72, 183-225 (2003)

3. Daru, V., Tenaud, C.: High order one-step monotonicity-preserving schemes for unsteady compressible flow calculations. Journal of Computational Physics 193(2), 563-594 (2004)

4. Descombes, S., Duarte, M., Dumont, T., Louvet, V., Massot, M.: Adaptive time splitting method for multi-scale evolutionary PDEs. Confluentes Mathematici (to app.) (2011)

5. Descombes, S., Massot, M.: Operator splitting for nonlinear reaction-diffusion systems with an entropic structure: Singular perturbation and order reduction. Numer. Math. 97(4), 667-698 (2004)

6. Duarte, M., Massot, M., Descombes, S., Tenaud, C., Dumont, T., Louvet, V., Laurent, F.: New resolution strategy for multi-scale reaction waves using time operator splitting, space adaptive multiresolution and dedicated high order implicit/explicit time integrators. Submitted to SIAM J. Sci. Comput., available on HAL (http://hal.archives-ouvertes.fr/hal-00457731) (2010)

7. Duarte, M., Massot, M., Descombes, S., Tenaud, C., Dumont, T., Louvet, V., Laurent, F.: New resolution strategy for multi-scale reaction waves using time operator splitting and space adaptive multiresolution: Application to human ischemic stroke. ESAIM Proc. (to app.) (2011)

8. Hairer, E., Wanner, G.: Solving ordinary differential equations II, second edn. Springer-Verlag, Berlin (1996). Stiff and differential-algebraic problems

9. Harten, A.: Multiresolution algorithms for the numerical solution of hyperbolic conservation laws. Comm. Pure and Applied Math. 48, 1305-1342 (1995)

10. Laverdant, A., Candel, S.: Computation of diffusion and premixed flames rolled up in vortex structures. Journal of Propulsion and Power 5, 134-143 (1989)

11. Müller, S.: Adaptive multiscale schemes for conservation laws, vol. 27. Springer-Verlag, Heidelberg (2003)

12. Strang, G.: On the construction and comparison of difference schemes. SIAM J. Numer. Anal. 5, 506-517 (1968)

The paper is in final form and no similar paper has been or is being submitted elsewhere. 